\title{
Contacto y servicios locales en Andalucía: el «efecto contextual» del lugar de residencia
}

\author{
Contact and local services in Andalusia: \\ the contextual effect of place of residence
}

\author{
Clemente J. NAVARRo YÁÑEZ \\ Fundación Centro de Estudios Andaluces \\ Universidad Pablo de Olavide \\ cnavyan@upo.es \\ Cristina Mateos Mora \\ Fundación Centro de Estudios Andaluces \\ Universidad Pablo de Olavide \\ ematmor@upo.es
}

Recibido: 20/02/2012

Revisado: 11/03/2012

Aceptado: 11/05/2012

Disponible on line: 03/08/2012

\section{Resumen}

El contacto entre ciudadanía y administración municipal es una forma de participación extendida y relevante, pues supone la transmisión de demandas e información sobre problemas y servicios en las ciudades, actuando, pues, la ciudadanía como coprovisores de servicios públicos. A través de una encuesta representativa de la población andaluza realizada en 2010, en este artículo se analiza la frecuencia del contacto de la ciudadanía andaluza, los rasgos socio-políticos de quienes lo hacen y las razones por las que contactan. Esto permite ofrecer una visión de la extensión de este fenómeno participativo, comúnmente poco estudiado, así como las razones que lo originan. En especial, se pretende mostrar la existencia de efectos contextuales derivados del tamaño municipal y su inserción en la dinámica de áreas metropolitanas.

Palabras clave: participación, contacto, efecto contextual, administración municipal, áreas metropolitanas.

\begin{abstract}
Contact between the public and public administration is a form of long-term, relevant participation since it assumes the transmittal of requests and information about problems and services in cities, making, in effect, the citizenry part of public service provision. By presenting a representative survey of the Andalusian population taken in 2010, this article analyzes the frequency of contact of the Andalusian public, their socio-political characteristics and their reasons for the contact. This permits a view of the extent of this rarely studied participatory phenomenon, as well as its causes. In particular, this article seeks to show the existence of contextual effects based on the size of the municipality and its participation in the dynamics of urban areas.
\end{abstract}

Key words: participation, contact, contextual effect, municipal administration, urban areas.

Referencia normalizada: Navarro Yáñez, C. J., y Mateos Mora, C. (2012): «Contacto y servicios locales en Andalucía: el «efecto contextual» del lugar de residencia». Cuadernos de Trabajo Social, 25(2): 321-332.

Sumario: Introducción: el contacto como modo de activismo político. 1. ¿Quién contacta? Recursos, necesidades, percepción de problemas y lugar de residencia. 2. Variaciones locales en la extensión del contacto en Andalucía: tamaño municipal y dinámica metropolitana. 3. El efecto contextual del urbanismo: percepción de problemas y lugar de residencia. 4. A modo de conclusiones: sobre las desigualdades territoriales de la participación política. 5. Referencias bibliográficas. 
Introducción: el contacto como modo de activismo político

Aunque bastante extendido entre la ciudadanía, el contacto con las autoridades y administraciones públicas suele ser un modo de activismo político que recibe menos atención que otros más clásicos, como el asociacionismo, u otros más novedosos o más presente en la agenda pública, como la protesta colectiva. En parte, se debe a que los recursos y actitudes que suelen explicar otros tipos de activismo no son tan relevantes para este modo participativo, así como también por el hecho de que suele entenderse que las demandas se basan en intereses particulares.

No obstante, supone un mecanismo muy importante de representación de intereses. Por un lado, porque aunque individual, quien lo ejerce representa o llama la atención sobre un problema que concierne al conjunto de los afectados por ello, que además suele referirse a la inexistencia de un servicio público o a su mal funcionamiento. De esta forma, la ciudadanía individualmente o quienes contactan, actúan como agentes co-provisores de servicios públicos, en la medida en que transmiten información sobre su necesidad o posibles mejoras (Mladenka, 1977). A diferencia de otras formas participativas centradas en entidades colectivas, suponen un proceso de representación abierta por el que un solo ciudadano hace llegar a los responsables públicos su interés, aparentemente particular, pero expresando con ello el interés de todo un colectivo de afectados, llamando así la atención sobre un asunto o un debate público (García de la Cruz, 1995). Tal y como reconocen Verba, Scholzman y Brady (1995), aunque el objeto principal de los contactos particularizados obedezcan a necesidades básicas personales, estas también pueden entenderse como información sobre el problema o servicio público al que se refieren (p.89). Suponen, un ejercicio de «voz» individual por el que pueden verse representados intereses colectivos. De ahí el interés de su estudio.

Este fenómeno es especialmente interesante cuando el foco del contacto son las autoridades y administraciones públicas municipales. Por un lado, por la extensión del fenómeno: a finales de los años 1980, el 10 por ciento de la población norteamericana había realizado un contacto particular con el municipio. En el caso español, por ejemplo, el 6 por ciento de los casi 4.000 entrevistados en ciudades intermedias españolas en 2006 había contactado con un concejal o con el alcalde, el 14 por ciento lo había hecho con un funcionario o empleado municipal, y el 9 por ciento había presentado una queja o reclamación ante el ayuntamiento. Representan tasas mayores que otras modalidades participativas referidas al ámbito local, como el contacto con una asociación o la participación en una manifestación (Navarro, Cuesta y Font, 2009). Por otro lado, porque se trata de ámbitos cercanos que facilitan el contacto e interacción entre ciudadanos y autoridades públicas. También, porque los municipios, aunque no los diseñen o tengan competencias directas sobre ellos, prestan una amplia gama de servicios importantes de cara a la calidad de vida de la ciudadanía. No obstante, existen diferencias importantes en el tamaño, composición o dinámica social, cultural y política de los municipios que pueden influir en la extensión de la participación, en general, y de este modo participativo en particular (Navarro, 2011).

¿Cuántos ciudadanos y ciudadanas contactan con sus ayuntamientos?, ¿quiénes son? o mejor, ¿qué factores explican que lo hagan? Estas serán las cuestiones a las que trataremos de responder en este artículo para el caso de la población andaluza. Para ello presentamos este análisis, utilizando la Encuesta sobre la Realidad Social Andaluza (ERSA) que realiza la Fundación Centro de Estudios Andaluces. En primer lugar presentamos algunos argumentos sobre la naturaleza del contacto como activismo político y sus factores explicativos. En segundo lugar, mostramos evidencias descriptivas sobre el efecto del municipio de residencia de los entrevistados y sobre su extensión. En tercer lugar, mediante análisis multivariable, mostramos la existencia de un efecto contextual derivado del municipio de residencia; finalizaremos el artículo con unas breves conclusiones.

Pero además de las razones expuestas, el análisis de estos efectos contextuales tiene interés en relación a la práctica profesional de la denominada Administración cercana (street level bureaucracy), tal y como la denominara Lipsky (1971), en la que se integra una buena parte de las trabajadoras y trabajadores sociales que desempeñan su labor en los munici- 
pios. Se trata de empleados municipales que trabajan en primera línea, en el momento de la producción directa de servicios a la ciudadanía y que por este factor suponen un actor que canaliza buena parte del activismo de contacto que desarrolla la ciudadanía. La cuestión es que el aumento de la actividad de contacto representa un incremento en el volumen de trabajo que ha de atender la Administración cercana, redundando en la necesidad de establecer «estrategias de contingencia» que van desde el establecimiento de reglas sobre la definición de diferentes tipos de demandas, a los recursos y estrategias de intervención necesarias para atenderlas. La cuestión es que estas reglas no suelen estar definidas institucionalmente, sino que se desarrollan como una parte más del desempeño profesional, redundando decisivamente en el grado de éxito de los servicios (Whitaker, 1980; Hudson, 1989). Estas reglas en uso que desarrollan los profesionales resolverán el «dilema de implementación» que supone la ausencia o indefinición de reglas específicas para la producción de servicios junto a la sobrecarga de demandas, más acuciada en aquellos contextos municipales donde exista mayor actividad de contacto (Navarro Rodríguez, 2009).

\section{1. ¿Quién contacta? Recursos, necesidades, percepción de problemas y lugar de residencia} Desde los primeros estudios sobre participación, el contacto ha recibido un tratamiento particular, tanto por su carácter estrictamente individual como por la escasa relación con los dos factores explicativos básicos de otros modos participativos: por un lado, los recursos individuales o estatus socioeconómico, y por el otro, el nivel de involucración política medida a través de actitudes políticas, tal y como muestran los análisis de diversos modos de activismo político para el caso español (Ferrer, Medina y Torcal, 2006). Por ello se ha indicado que el contacto surge en un proceso diferente del que rige para otras modalidades participativas (Verba y Nie, 1972, p. 135). La idea sería que, dado su marcado carácter individual e instrumental, sobre todo cuando se trata de contacto particularizado (sobre problemas personales), subyace una lógica participativa diferente de la de otros modos participativos. Pero, ¿no debería, estar relacionado con el estatus socioeconómico?, ¿no deberían hacerlo en mayor medida quienes ne- cesitan mayor actuación pública? El hecho es que los estudios suelen mostrar una baja asociación entre el contacto, el estatus socioeconómico y las actitudes políticas; al menos, una asociación más baja que para otros modos participativos (Mladenka, 1977; Sharp, 1984; Velditz, Dyer y Durand, 1980; Verba y Nie, op. cit.).

De ahí que, ni la clásica tesis socioeconómica, que supone una asociación positiva entre recursos individuales y participación, ni su contraria, la tesis de las necesidades, parecen aportar buenas explicaciones respecto al contacto. En este sentido, la literatura ha ido proponiendo dos argumentos explicativos. En primer lugar, la tesis de la relación entre la necesidad y la competencia política (Jones, Greenberg, Kaufman y Drew, 1977) supone que, aunque los recursos individuales o familiares puedan estar relacionados con las necesidades o las actitudes políticas, tal y como sostiene la tesis socioeconómica (Verba y Nie, 1972), el contacto, al ser un modo participativo individual y centrado en problemas concretos, se produce cuando existe cierta combinación de esos dos factores. Además de tener un problema derivado de su situación socioeconómica, el ciudadano debe haber solventado las barreras que suponen el desconocimiento de las vías de transmisión de las demandas o las relativas a actitudes que desincentiven su actuación, como son: el sentimiento de ineficacia política, la resignación o el miedo al enfrentamiento (García de la Cruz, 1995, p. 55). Por lo tanto, a estatus superior corresponden menos necesidades y aumenta el sentimiento de competencia política, pero es poco probable que haya motivos para contactar con las autoridades públicas municipales. Al contrario, a estatus inferior corresponde un incremento de las necesidades, pero se reduce el sentimiento de eficacia política, por lo que es poco probable que estos ciudadanos crean que sus demandas serán atendidas. De ahí que el contacto se dará entre aquellos que poseen, a la vez, ciertas necesidades y cierta competencia política subjetiva. No obstante, la literatura muestra que, en general, existe una escasa relación entre problemas objetivos en los barrios o las necesidades objetivas de los ciudadanos con el contacto, sobre todo, si estas se miden a través de sus ingresos (Thomas, 1982).

La segunda tesis sostiene que el factor explicativo principal es, sobre todo, la percepción 
de problemas y necesidades por encima del de su existencia objetiva. Las necesidades pueden surgir por la percepción sobre distintos problemas o servicios municipales con independencia del estatus (Serra, 1995; Thomas y Melkers, 1999), incluso aumentan entre quienes tienen un estatus más elevado, porque sus preferencias o necesidades son nuevas o distintas, tal y como apuntan, por ejemplo: la tesis postmaterialista (Inglehart, 1998), el cambio hacia una nueva cultura política (Clark y Navarro, 2007), o la argumentación de Thomas (1982) respecto a los servicios municipales. Así, diversos estudios muestran que la percepción sobre problemas o sobre diferentes áreas de servicios municipales suele explicar mejor el contacto que el estatus o la existencia de necesidades objetivas (Sharp, 1984; Thomas, 1982; Traut y Emmert, 1993).

No obstante, además de esos factores individuales, la literatura también ha destacado el papel que en el desarrollo del contacto desempeña el municipio en el que se reside, al configurar un contexto socio-político, un conjunto de oportunidades que incentivan o inhiben el activismo participativo, y el contacto con las autoridades públicas en particular. A este respecto existen dos grandes perspectivas o tesis: por un lado, la tesis comunitaria que mantiene su principal argumento en el hecho de que un mayor tamaño poblacional está unido a una menor cercanía entre las administraciones y la ciudadanía y, por lo tanto, que aumenta el esfuerzo que requiere la participación, en particular, aquellos modos participativos de carácter comunitario que implican la interacción entre ciudadanos (como el asociacionismo) o el contacto de éstos con las autoridades públicas. Por otro lado, la tesis de la movilización que propone el efecto contrario: en contextos de mayor tamaño existe mayor diversidad entre los intereses de los ciudadanos lo que provoca un mayor activismo participativo. La cuestión es que estas tesis, aunque opuestas en relación al efecto de la dimensión del municipio de residencia, señalan la importancia del contexto comunitario en donde residen los ciudadanos en relación con la participación.

Ahora bien, en este caso el tamaño es considerado como un indicador de un fenómeno más amplio, como es el urbanismo. No se trata únicamente de un mayor volumen de pobla- ción, sino de su diversidad, o de estar más o menos en contacto con la dinámica urbana que caracteriza a las grandes ciudades. Desde esta última perspectiva, se trataría más bien del fenómeno de las áreas metropolitanas y la suburbanización. Así, se indica que en este espacio suele situarse el locus característico del «declive cívico», en la medida en que el commuting que implica el proceso de suburbanización aleja o hace que se pierda interés por los problemas del municipio en el que únicamente se reside, y se desarrolle otro conjunto de actividad principales en la ciudad central (trabajo, consumo o uso de servicios públicos), o simplemente, porque el desplazamiento cotidiano que implica el commuting supone un coste de tiempo que reduce el que pueda destinarse a la participación (Putnam, 2000).

Verba y Nie (1972) muestran para el caso norteamericano que el contacto es menor en los municipios sujetos a esta dinámica urbana, y en cambio es mayor en las ciudades de tamaño medio que no están sujetas a esa dinámica. En general, cuando extienden su estudio a otros países, parece confirmarse la diferencia entre el ámbito rural y el urbano, a favor del primero (Verba, Nie y Kim, 1978). Del mismo modo, Oliver (2001) muestra que a mediados de los años 1990 el contacto aumenta cuando disminuye el tamaño municipal, con cierta independencia de que pertenezca o no al área metropolitana. Para el caso español, y con datos de 2002, Navarro (2008) muestra que el tamaño y la pertenencia a un área metropolitana cuentan para explicar la propensión de la población a la realización del contacto: ambas características cuentan negativamente, sobre todo en los municipios de mayor tamaño (más de 50.000 habitantes) que se integran en un área metropolitana. En general, el porcentaje de población que se involucra en esta forma de activismo decae cuando vive en municipios urbanos, sea por su tamaño o su pertenencia a un área metropolitana (Navarro, 2011).

Este fenómeno supone que, además de los recursos, las actitudes y la percepción de problemas, el contacto es un modo participativo sujeto a efectos contextuales derivados del urbanismo que caracteriza al municipio de residencia; es decir, existe una ecología territorial del contacto que, sobre todo, se ha de poner en relación con el coste que implica el desarrollo de 
este modo de activismo político, ya que aumenta el esfuerzo que significa el aumento de la distancia entre ciudadanía y autoridades públicas que se deriva de la escala de la comunidad local.

En este trabajo pretendemos mostrar evidencias de la existencia de esa ecología del contacto en el caso de los municipios andaluces mediante el análisis de una encuesta representativa realizada en el año 2010. En concreto, pretendemos mostrar, en primer lugar, si el tamaño del municipio y el hecho de que éste se integre en un área metropolitana da cuenta de diferencias en el porcentaje de entrevistados que contactaron con la Administración municipal. Y en segundo lugar, si ese efecto contextual existe con independencia de la influencia de factores de índole individual, sean los recursos de los entrevistados, sus actitudes políticas $\mathrm{o}$, en especial, su percepción de los problemas existentes en su municipio: así como la relación que pueda existir entre este último aspecto y el tipo de municipios (según tamaño o su carácter metropolitano). En suma, el objetivo principal de este trabajo consiste en mostrar si existe un efecto contextual ligado al carácter urbano del municipio en el que reside la ciudadanía.

\section{Variaciones locales en la extensión del contacto en Andalucía: tamaño municipal y dinámica metropolitana}

¿Cuántos andaluces y andaluzas contactan con sus ayuntamientos?, ¿qué factores lo explican? $\mathrm{y}$ en particular, ¿existe un efecto contextual como el que parecen mostrar evidencias para España y otros países? Para responder a estas preguntas analizaremos la Encuesta sobre la Realidad Social Andaluza (ERSA), realizada en 2010 que ofrece información para analizar este asunto, en particular se trata de una muestra de 1.203 entrevistas, distribuidas proporcionalmente según provincias y tamaño municipal (según número de habitantes). Bajo supuesto de muestreo aleatorio simple, máxima variabilidad y nivel de confianza del 95,5 por ciento, el error es del 2,8 por ciento para la muestra en su conjunto. En concreto, se pregunta a los entrevistados si contactaron con sus ayuntamientos durante los últimos doce meses: alrededor de un 10 por ciento declara haberlo hecho, lo que supone que alrededor de 650.000 andaluces y andaluzas realizaron algún tipo de contacto con su Administración más cercana, los

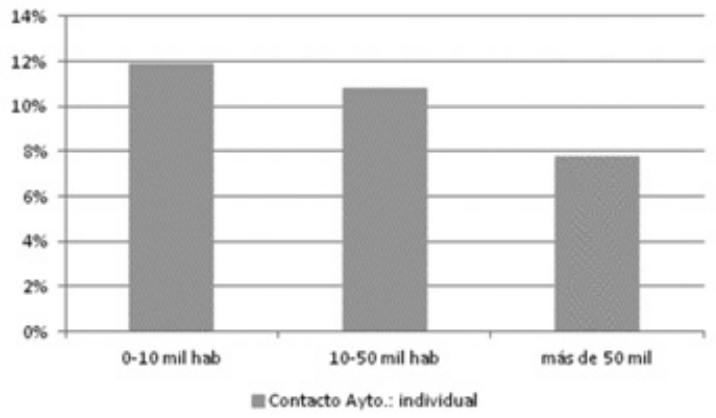

Gráfico 1. Contacto individual según tamaño del municipio.

Fuente: Elaboración propia a partir de datos del ERSA 2011.

Nota: Las diferencias son significativas para $\mathrm{p}<0,05$.

ayuntamientos (Anexo 1). Pero, ¿existen diferencias según las características de los municipios en los que residen?

En el Gráfico 1 puede apreciarse claramente la pauta señalada previamente: cuanto mayor es el tamaño del municipio de residencia de los entrevistados, en menor proporción contactaron con sus ayuntamientos los últimos doce meses. Cabe señalar, como sostiene la tesis de la pérdida de comunidad, el tamaño del municipio implica diferentes costes para el desarrollo de este modo de activismo político.

Pero, iinfluye en la extensión del contacto el hecho de residir en un área metropolitana? Según Ferias (2010), en Andalucía existen 10 áreas metropolitanas, además de la ciudad central, son municipios metropolitanos de diverso tamaño, y en los que al menos el 10 por ciento de su fuerza de trabajo realiza commuting, esto es, se desplaza a otro municipio diferente del de residencia para desempeñar su actividad laboral. A partir de esta delimitación, hemos generado una variable de la dinámica metropolitana en la que residen los entrevistados, en concreto, diferenciando tres tipos de municipios: ciudad central, municipio metropolitano y municipio no metropolitano. Tal y como puede apreciarse en el Gráfico 2, son los municipios que no se encuentran integrados en un área metropolitana los que presentan un mayor porcentaje de contacto entre sus habitantes (12 por ciento), siendo los grandes núcleos o ciudades centrales aquellos en los que se da en menor medida el fenómeno (7 por ciento). Dado que entre los municipios metropolitanos y no metropolitanos los hay de 


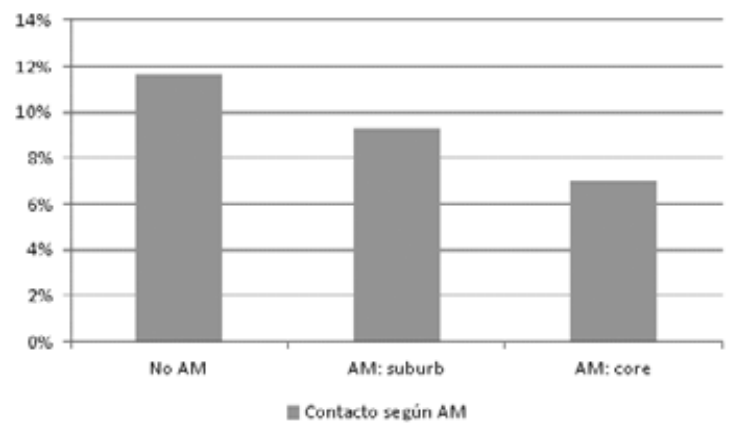

Gráfico 2. Contacto según dinámica metropolitana. Fuente: Elaboración propia a partir de datos del ERSA 2011.

Nota: Las diferencias son significativas para $\mathrm{p}<0,05$.

diverso tamaño, cabría señalar que parece existir un «efecto metropolitano» sobre el contacto con los ayuntamientos diferente del «efecto tamaño» municipal.

Cabría pensar, como muestran algunos de los análisis mencionados anteriormente, que existe un efecto integrado de tamaño y dinámica metropolitana. En concreto, a partir de los resultados recién presentados se puede pensar que en los municipios de dimensiones más pequeñas, especialmente si no están situados en un área metropolitana, es en donde está más extendido el contacto con sus respectivos ayuntamientos. Para poder constatarlo hemos creado una tipología de municipios que cruza los dos criterios (tamaño y dinámica metropolitana). Tal y como muestra el Gráfico 3, el contacto está más extendido entre los municipios no metropolitanos, con cierta independencia de su tamaño: tanto los municipios de tamaño medio (de 10.000 a 50.000 habitantes, como en los grandes (más de 50.000 habitantes) el contacto es mayor si no se trata de municipios metropolitanos. Al contrario, se destaca la extensión del fenómeno del contacto en los municipios más pequeños que están en un área metropolitana: en este tipo de municipio el contacto alcanza los niveles más elevados (28,6 por ciento).

Básicamente, este tipo de municipios, más que corresponder al típico suburb de tipo residencial que caracteriza el proceso de suburbanización, constituyen espacios rurales de pequeño tamaño que, aunque han crecido en población, se han incorporado a la dinámica metropolitana por la involucración de sus trabajadores en

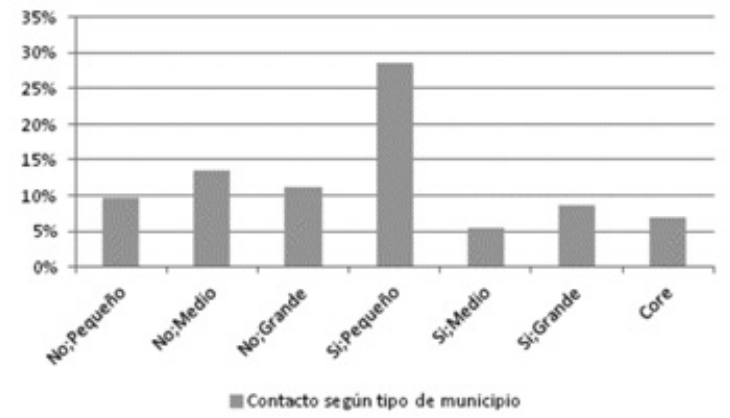

Gráfico 3. Contacto según tipo de municipios (tamaños y dinámica metropolitana).

Fuente: Elaboración propia a partir de datos del ERSA 2011.

Nota: Las diferencias son significativas para $\mathrm{p}<0,05$.

el mercado de trabajo metropolitano (normalmente de la gran ciudad), sin que en ellos se haya producido un cambio sustantivo de los patrones sociales y culturales, básicamente de tipo comunitario (Navarro, 2011). No obstante, también es cierto que su inclusión en un área metropolitana ha supuesto algunos cambios relevantes, tanto por la llegada de nuevos habitantes, normalmente familias jóvenes con hijos, como por la necesaria adaptación de su trama urbana o las nuevas necesidades de regulación de tráfico o de transporte con otros municipios, principalmente, la ciudad central.

Así, aunque en términos absolutos los cambios no adoptan las dimensiones que toman en otros municipios más grandes y previamente ligados a la ciudad central, en términos relativos pueden ser ampliamente sentidos por sus habitantes. Esto es, la percepción de los problemas derivados de su involucración en la dinámica metropolitana por parte de sus residentes puede ser mayor que en otros tipos de municipios de mayor tamaño o en los que es menos reciente la integración en la dinámica metropolitana. En este sentido, tal y como hemos indicado más arriba, la literatura sobre el contacto, sin desatender el efecto que en este tipo de comportamiento participativo pueden tener los recursos y actitudes socio-políticas individuales, señala la importancia de la percepción de problemas como motivo principal para la implicación en este modo de activismo político. ¿Perciben más problemas los habitantes de estas pequenas comunidades metropolitanas que los de otros tipos de municipios? 
A los entrevistados se les preguntó si consideraban que en su barrio existían diferentes problemas, que en términos generales podían agruparse en cuatro tipos básicos, a saber: la inseguridad, los relativos a la urbanización o condiciones materiales de la ciudad (mal estado o suciedad de las calles, tráfico o malas comunicaciones), los relacionados con el medio ambiente (ruidos, malos olores, ausencia o mal estado de zonas verdes) y con los servicios personales (socio-culturales, para la infancia o para los mayores) (Anexo 2). Hemos elaborado un indicador con recorrido $(0,1)$, en donde el cero significa que no se menciona ningún problema y 1 que se mencionan todos los incluidos en cada tipo, o bien, todos en su conjunto cuando así los consideramos. ¿En qué tipo de municipios se percibe más problemas? Y ¿de qué tipo concreto?

En la Tabla 1 se aprecia, para la muestra en su conjunto, que la inseguridad es el problema menos mencionado, siendo la situación bastante equilibrada para los otros tres tipos de problemas. Ahora bien, su pauta es diferente según el tipo de municipios. Así, aquélla se menciona en mayor medida en las grandes ciudades de las áreas metropolitanas o sus núcleos, y aunque estos tipos de municipios también destacan en los otros tipos de problemas, se señalan en mayor medida por los entrevistados que residen en municipios pequeños de las áreas me- tropolitanas. Esto tiene sentido si se considera que no son los mismos problemas los que pueden percibir los habitantes de un municipio de gran tamaño de un área metropolitana que los de un municipios de menor tamaño: en éstos existen, en mayor medida, o al menos son más perceptibles, por el cambio que ha supuesto su involucramiento metropolitano, el aumento del tráfico, de la contaminación o la demandas de servicios para la población de más edad o los nuevos residentes con menores. Por ejemplo, la distribución de los presupuestos en los municipios y los Servicios Sociales en particular, se organizan con criterios relativos al tamaño municipal, con independencia de la dinámica metropolitana y sus posibles efectos. Este reciente fenómeno puede explicar que para sus habitantes la percepción de problemas sea mayor que para otros municipios más grandes del área metropolitana y, por supuesto, que para aquellos municipios de igual tamaño que no se han integrado en la dinámica metropolitana. En general, puede apreciarse que es en este tipo de municipio donde existe un mayor número de percepción de problemas: el 32 por ciento de todos los propuestos.

\section{El efecto contextual del urbanismo: per- cepción de problemas y lugar de residencia} Evidentemente, además de los rasgos contextuales de los municipios, sus residentes se ca-

\begin{tabular}{|l|l|c|c|c|c|c|}
\hline $\begin{array}{c}\text { iÁrea } \\
\text { metropolitana? }\end{array}$ & Tamaño municipal & Inseguridad & Urbanización & $\begin{array}{c}\text { Medio } \\
\text { ambiente }\end{array}$ & $\begin{array}{c}\text { Servicios } \\
\text { personales }\end{array}$ & Total \\
\hline \multirow{2}{*}{ No } & Pequeño (>10 mil) & 0,12 & 0,55 & 0,42 & 0,52 & 0,22 \\
\cline { 2 - 7 } & Medio (10-50 mil) & 0,18 & 0,49 & 0,38 & 0,46 & 0,22 \\
\cline { 2 - 7 } & Grande (>50 mil) & 0,11 & 0,58 & 0,71 & 0,53 & 0,25 \\
\hline \multirow{2}{*}{ Sí } & Pequeño (<10 mil) & 0,04 & $\mathbf{0 , 8 9}$ & $\mathbf{0 , 7 4}$ & $\mathbf{0 , 6 3}$ & $\mathbf{0 , 3 2}$ \\
\cline { 2 - 7 } & Medio (20-50 mil) & 0,19 & 0,52 & 0,39 & 0,58 & 0,23 \\
\cline { 2 - 7 } & Grande (>50 mil) & $\mathbf{0 , 2 6}$ & 0,56 & 0,53 & 0,59 & 0,30 \\
\cline { 2 - 7 } & Ciudad central & 0,21 & 0,59 & 0,56 & 0,49 & 0,27 \\
\hline \multirow{2}{*}{ Total } & 0,19 & 0,56 & 0,491 & 0,52 & 0,25 \\
\hline
\end{tabular}

Tabla 1. Problemas según tipos de municipios. Medias en escalas $(0,1)$.

Fuente: Elaboración propia a partir de datos del ERSA 2011.

Nota: Diferencias significativas para $\mathrm{p}<0,05$, salvo para los servicios personales $(\mathrm{p}<0,10)$, habiéndose utilizado el estadístico F de Snedecor. 
racterizan por la posesión de diferentes recursos individuales y actitudes políticas que la literatura señala que suelen explicar el activismo político. Ya hemos indicado que en el caso concreto del contacto, los primeros no suelen ser factores muy explicativos que las actitudes políticas, y especialmente, la percepción de problemas. Cabría pensar, pues, que los recursos, y especialmente, las actitudes y la percepción de problemas permitirían explicar el contacto en mayor medida que el contexto municipal. Esto es, que existe un «efecto composición» derivado del modo de ser de los residentes, de sus actitudes y percepciones, sin que exista un «efecto contextual» relacionado con el tipo de municipio en el que residen.

Para tratar de aclararlo hemos realizado modelos de regresión logística que incluyen, además de nuestra tipología de municipios, algunas variables que suelen explicar los comportamientos participativos, referidas tanto a recursos (edad, género o estudios), como a actitudes políticas (valoración de servicios municipales, sentimiento de eficacia política o interés por la política), así como también el número de problemas mencionados sobre el total de los propuestos en la encuesta (Anexo 3). ¿Existe un efecto contextual derivado del tipo de municipio en el que residen los entrevistados?

Los resultados, que se presentan en la Tabla 2 , muestran que los factores que tradicionalmente suelen explicar el contacto también aparecen en nuestro análisis, a saber: la percepción subjetiva de los ingresos (cuando se considera que se llega difícilmente a final de mes), el interés por la política y la percepción de problemas en el barrio. Pero además, con independencia de estos factores, el tipo de municipio influye en la probabilidad de que un entrevistado o entrevistada contacte con su ayuntamiento. En concreto, hemos escogido como categoría de referencia de la variable tipo de municipio la ciudad central de las áreas metropolitanas, por tanto, en comparación con éstas, que es donde en menor medida se da el contacto, son los municipios de tamaño medio y pequeño que no pertenecen a un área metropolitana los que más se diferenciando de ellas. Pero destacan, sobre todo, los entrevistados que viven en municipios pequeños de áreas metropolitanas. Por tanto, si el tamaño, cuando es reducido, incentiva el contacto, lo hace especialmente cuando se trata de comunidades que por integrarse en áreas metropolitanas enfrentan más, nuevos o diferentes problemas que los municipios del mismo tamaño que no están integrados en un área metropolitana. En este sentido, Navarro (2011) muestra que para el conjunto de España el contacto se produce especialmente en comunidades pequeñas pero heterogéneas en su composición social, aquellas en donde es más susceptible que aparezcan conflictos derivados de la recepción de determinados servicios.

\section{A modo de conclusiones: sobre las des- igualdades territoriales de la participación política}

Los resultados presentados muestran que el contacto está sujeto a la influencia de efectos contextuales derivados del lugar en el que reside la ciudadanía. En sintonía con los resultados previos, que confirman la tesis de la pérdida de comunidad, el contacto es una actividad más extendida entre ciudadanía que reside en municipios más pequeños, pero también, entre aquellos que no están integrados en un área metropolitana. Ambos factores juegan como una estructura de oportunidades que incrementa - o disminuye - el esfuerzo que requiere un modo de activismo que, por ser individual, requiere una fuerte implicación personal. De otra forma, la existencia de este efecto contextual supone que, a igualdad de otros factores, como los recursos, las actitudes o la percepción de problemas, existen más ciudadanos que actúan como «sujetos intensos» cuando no tienen que enfrentar a la barrera que supone el tamaño y/o la lógica metropolitana.

Esta es, claramente, la pauta general. No obstante, también hemos evidenciado que cuando el coste es más bajo, por ejemplo en los municipios más pequeños y la percepción de problemas es mayor, se produce la tasa de contacto más elevado. Se trata de municipios pequeños que se integran en un área metropolitana. Esto mostraría que el contacto depende más de la percepción de problemas, que de los recursos y actitudes políticas individuales, pero que aquélla a su vez, se ve influida por el tipo de municipio en el que se reside. Así, en línea con los argumentos de la tesis de la movilización, el urbanismo que deriva de la dinámica metropolitana, incentiva el activismo político porque existen más problemas que movilizan a la ciu- 


\begin{tabular}{|l|c|c|c|}
\hline & Coeficiente & Error & Wald \\
\hline Género (varón=1) & $-0,238$ & 0,242 & 0,968 \\
\hline Edad & $-0,271^{*}$ & 0,123 & 4,822 \\
\hline Nivel de estudios & $-0,013$ & 0,143 & 0,008 \\
\hline Percepción ingresos hogar & 0,101 & 0,152 & 0,447 \\
\hline Eficacia política & $-0,077$ & 0,058 & 1,758 \\
\hline Evaluación servicios municipales & 0,022 & 0,079 & 0,079 \\
\hline Interés política municipal & $2,014 * *$ & 0,414 & 23,660 \\
\hline Confianza en el ayuntamiento & $-0,095$ & 0,065 & 2,141 \\
\hline Percepción problemas & $1,851^{* *}$ & 0,619 & 8,930 \\
\hline Municipio: no metropolitano, $<10$ mil & $0,804^{*}$ & 0,362 & 4,942 \\
\hline Municipios: no metropolitano, $50-100$ mil & $0,987^{* *}$ & 0,344 & 8,215 \\
\hline Municipios: no metropolitano, $>100$ mil & 0,739 & 0,515 & 2,065 \\
\hline Municipio: metropolitano, $<10$ mil & $1,762^{* *}$ & 0,536 & 10,805 \\
\hline Municipios: metropolitano, 10-50 mil & 0,021 & 0,486 & 0,002 \\
\hline Municipios: metropolitano, $>100$ mil & 0,171 & 0,408 & 0,176 \\
\hline Constante & $-2,875^{* *}$ & 0,820 & 12,283 \\
\hline R2 de Nagalkerke & 0,139 & & \\
\hline Casos correctamente clasificados & $909(90,4 \%)$ & & \\
\hline
\end{tabular}

Tabla 2. El contacto con el ayuntamiento: recursos, actitudes políticas, percepción de problemas y tipos de municipios. Regresión logística.

La categoría de referencia para la tipología de municipios es la «ciudad central».

Significatividad: $* \mathrm{p}<0.05, * * \mathrm{p}<0.005$.

dadanía. El contacto, no se da sobre todo cuando existen menos costes (municipios pequeños), sino cuando a esto se suma la existencia de problemas que derivan del proceso de suburbanización, o mejor dicho, de la percepción de los residentes de municipios, que constituyen comunidades locales tanto desde una perspectiva socio-demográfica, como por sus formas de vida características.

En general, por lo expuesto se supone que el lugar de residencia aparece como una estructura de oportunidades que, con cierta independencia de características individuales, crea desigualdades en las posibilidades que tienen los ciudadanos y ciudadanas para transmitir sus demandas a las autoridades públicas de forma individual. No se trata tanto, o únicamente, de que las diferencias sociales se traduzcan en desigualdades políticas, sino que en el caso del contacto, su ecología municipal también se convierte en desiguales oportunidades de participación política. Esto supone que en el análisis de la participación, como una actividad no sólo deseable normativamente, sino también en relación a la detección de nuevas necesidades o mejoras en el funcionamiento de los servicios públicos, debe considerarse como un factor más el lugar en el que residen los habitantes, pues implica la existencia de efectos contextuales 
que generan ciertas desigualdades, diferentes o añadidas a las derivadas de la desigualad social, ya ampliamente conocidas.

Por otra parte, y desde la perspectiva del ejercicio profesional a la que hemos denominado anteriormente Administración cercana y en la que se integraría buena parte de las trabajadoras y trabajadores sociales que desempeña su labor en los municipios, esto podría implicar que allí donde suelen existir menos recursos y personal, quizás exista un mayor volumen de de demanda, al menos, por lo que se refiere a la extensión del contacto. Indudablemente, tal y como señala la literatura sobre la Administración cercana, este factor redunda significativamente en las estrategias de intervención que han de desarrollar, y en concreto, en las estrategias adaptativas en cuanto a la definición de las demandas, así como los recursos y los métodos de intervención a utilizar para atenderlas. Desgraciadamente, no contamos en este trabajo con información detallada sobre estos procesos, pero sin duda reclama la necesidad de contextualizar también el marco territorial en el que las trabajadoras y trabajadores sociales desempeña su labor, si se quiere, como sugerencia para otros estudios, el análisis de la que tentativamente pudiéramos denominar «ecología del desempeño profesional del Trabajo Social».

\section{Referencias bibliográficas}

Clark, T.N. y Rempel, M. (eds.) (1997). Citizen Politics in Post-Industrial Societies. Bulder, Co: Westview Press.

Clark, T.N. y Navarro, C.J. (eds.) (2007). La nueva cultura política. Tendencias globales y casos iberoamericanos. Buenos Aires: Miño y Dávila.

Coulter, Ph.B. (1988). Political Voice. Citizen demand for urban public services. Tuscalosa: The University of Alabama Press.

Feria, J.M. (2010). Movilidad residencial y los procesos de urbanización metropolitanos en España. En J.M. Feria y J.M. Albertos. (eds.), La ciudad metropolitana en España: procesos urbanos en los inicios del siglo XXI. Madrid: Thompson Reuters.

Ferrer, M. Medina, L. y Torcal, M. (2006). La participación política: factores explicativos, en J.R Montero, J, Font, y M. Torcal. (eds.), Ciudadanos, asociaciones y democracia en España (pp. 133-156). Madrid: CIS.

García de la Cruz, J.J. (1995). La defensa de intereses ante las autoridades públicas. ¿Acción colectiva o acción individual?. Revista Española de Sociología, 69, 39-68.

Hudson, B. (1989). Michael Lypsky and street level bureaucracy: a neglected perspective. En L. Barton (ed.), Disability and Dependency. Londres: Falmer Press.

Inglehart, R. (1998). Modernización y postmodernización. El cambio cultural, económico y político en 43 sociedades, Madrid: CIS.

Jones, B., Greenberg, S., Kaufman, C. y Drew, J. (1977). Bureaucratic response to citizen-initiated contacts: Environmental enforcement in Detroit. American Political Science Review, 71, 148-165.

Lipsky, M. (1971).Street-level bureaucracy and the analysis of urban reform. Urban Affairs Quarterly, 6, 391-409.

Mladenka, K. (1977). Citizen demand and bureaucratic response: Direct dialling democracy in a major American city. Urban Affairs Quarterly, 12, 273-290.

Montero, J.R., Gunther, R. y Torcal, M. (1998). Actitudes hacia la democracia en España: legitimidad, descontento y desafección. Revista Española de Sociología, 83, 9-49.

Navarro, C.J. (2006). La administración cercana. Técnicos municipales, descentralización y municipios. Córdoba: Ayuntamiento de Córdoba.

Navarro, C.J. (2008). Participación ciudadana y territorio: exploraciones para el caso español. Ciudad y Territorio. Estudios Territoriales, 156, 261-271.

Navarro, C.J. (2011). Comunidades locales y participación política en España. Madrid: CIS.

Navarro; C.J. y Pérez Yruela, M. (2002). La cultura política de los andaluces. En E. Moyano (ed.), La Sociedad Andaluza (pp.107-123). Córdoba: CSIC.

Navarro, C.J. y Clark, T.N. (julio, 2009). Contextualizing political participation. Territorial and institutional effects. IPSA World Conference, Santiago de Chile. 
Navarro, C.J. y Rodríguez, M.J. (2009): Gobernanza multi-nivel y sistemas locales de bienestar en España, Valencia, Tirant lo Blanch.

Navarro, C.J., Cuesta, M. y Font, J. (2009). ¿Municipios participativos? Participación política y ciudadana en ciudades medias españolas. Madrid: CIS.

Oliver, J.E. (2001). Democracy in Suburbia. Princeton: Princeton University Press.

Putnam, R. (2000). Bowling Alone. Nueva York: Basic Books.

Serra, G. (1995). Citizen-initiated contacts and satisfaction with bureaucracy: A multivariate analysis. Joural of Public Administration Theory and Research, 5, 175-178.

Sharp, E.B. (1982). Citizen initiated contacting and social status: Determining the relationship and accounting for it. American Political Science Review, 76, 109-115.

Sharp, E.B. (1984).Citizen-Demand Making in Urban Context. American Journal of Political Science, 28, 654-670.

Thomas, J.C. (1982). Citizen-initiated contacts with governments agencies: A test of three theories. American Journal of Political Science, 26, 504-522.

Thomas, J.C. y Melkers, J. (1999). Explaining citizen-initiated contacts with municipal bureaucrats. Lessons from the Atlanta Experience. Urban Affairs Review, 34 (5), 667-690.

Torcal, M.; Montero; J.R. y Toerell, J. (2006). La participación política en España: modos y niveles en perspectiva comparada. En J.R. Montero , J. Font y M Torcal,(eds.), Ciudadanos, asociaciones y participación en España (pp.47-76). Madrid: CIS.

Traut, C.A. y Emmert, C.F. (1993). Citizen-initiated contacting: a multivariable analysis. American Politics Quarterly, 12, 239-253.

Velditz, A.; Dyer, J.A. y Durand, R. (1980). Citizen contacts with local governments: A comparative view. American Journal of Political Science, 24, 50-67.

Verba, S. y Nie, N. (1972). Participation in America: Political democracy and social quality. Nueva York: Harper and Row.

Verba, S., Nie, N.H. y Kim, J. (1978). Participation and Political Equality. A Seven-Nation Comparison. Chicago: The University of Chicago Press.

Verba, S., Scholzman, K.L. y Brady, H.E. (1995). Voice and Equality. Civic Voluntarism in American Politics. Cambridge: Harvard University Press.

Whitaker, G.P. (mayo-junio, 1980). Coproduction: Citizen, Participation in Service Delivery. Public Administration Review, 70 (2), 240-246.

\section{ANEXO}

\section{Guión de las entrevistas}

Se especifican las preguntas usadas para elaborar los ítems que se utilizan en el artículo, así como, en el caso necesario, la forma en que han sido elaborados. Todos los indicadores se han estandarizado en escala $(0,1)$, desde su valor mínimo (que se ha hecho igual a 0) a su valor máximo (que se ha hecho igual a 1).

\section{Contacto con el ayuntamiento}

Durante los últimos doce meses, ¿ha contactado con un político, un funcionario del ayuntamiento?

$$
\text { 1. Sí 2. No 9. NC }
$$

\section{Percepción de problemas}

¿Diría que en su barrio o vecindario existe...? (RESPUESTA MÚLTIPLE. Anotar todos los que diga el entrevistado)
- Inseguridad ciudadana, delincuencia o vandalismo.

- Poca limpieza de las calles.

- Pocas zonas verdes o en mal estado.

- Pocos servicios o actividades para los niños/as.

- Pocos espacios o actividades culturales y deportivas.

- Problemas de tráfico o malas comunicaciones con el transporte público.

- Pocos servicios y actividades para las personas mayores.

- Ruidos exteriores, en la calle.

- Contaminación o malos olores provocados por industria, tráfico,...

- Otros problemas, ¿cuáles? 
- 98. Ningún problema, no existen problemas en mi barrio o vecindario.

- 99. NC.

Los ítems han sido agrupados en los tipos de problemas que se detallan a continuación. Para cada uno de ellos se ha elaborado un indicador con recorrido $(0,1)$, donde cero significa que no se menciona ninguno y 1 que se mencionan todos los incluidos ne cada tipo. El indicador sintético de percepción de problemas, utilizado en el análisis de regresión logística, sigue la misma lógica pero considerando todos los problemas $(0=$ ningún problema, $1=$ menciona todos los problemas).

- Inseguridad.

- Urbanización o condiciones materiales de la ciudad: mal estado o suciedad de las calles, tráfico o malas comunicaciones.

- Medio ambiente: ruidos, malos olores, ausencia o mal estado de zonas verdes.

- Servicios personales (socio-culturales, para la infancia o para los mayores).

\section{Actitudes políticos locales}

\subsection{Calidad servicios municipales}

$\mathrm{Y}$, en general, ¿como calificaría la calidad de los servicios en su barrio, de la zona donde vive? Utilice una escala donde 1 significa que la calidad de los servicios es pésima y 10 significa que son excelentes.

$\begin{array}{rrrrrrrrr}01 & 02 & 03 & 04 & 05 & 06 & 07 & 08 & 09 \\ 10 & 98 . & \text { NS } & 99 . & \text { NC } & & & & \end{array}$

\subsection{Interés por la política municipal}

Diría Ud. que la política municipal le interesa mucho, bastante, poco o nada
1. Mucho
2. Bastante
3. Poco
4. Nada
8. NS
9. $\mathrm{NC}$

\subsection{Eficacia política}

Media de las respuestas a los dos ítems que se muestran a continuación
Ahora le voy a leer una serie de afirmaciones y le pido que responda si está de acuerdo con cada una de ellas. Utilice, por favor, esta escala donde 1 significa que está totalmente en desacuerdo y 10 que está totalmente de acuerdo:

- La gente normal tiene poca capacidad para influir en las decisiones del ayuntamiento

- Los políticos locales no se preocupan mucho por lo que piensa la gente como yo

\subsection{Confianza institucional}

¿En qué medida confía Ud. en cada una de las siguientes instituciones o grupos? Utilice para ello la siguiente escala donde 1 significa ninguna confianza y 10 total confianza

El Ayuntamiento.

\section{Recursos individuales}

\subsection{Percepción ingresos hogar}

Pensando en la siguiente escala, ¿cuál es la capacidad de su hogar para llegar a fin de mes?

1. Con mucha dificultad

2. Con dificultad

3. Con cierta dificultad

4. Con cierta facilidad

5. Con facilidad

6. Con mucha facilidad

9. $\mathrm{NC}$

\subsection{Nivel de estudios}

¿Podría decirme cuál es su nivel de estudios terminados más alto?

- No sabe leer ni escribir $\quad 1$

- Inferior a Primaria 2

- Educación primaria 3

- Educación secundaria de $1^{\mathrm{a}}$ etapa 4

- Bachillerato 5

- Formación Profesional I 6

- Formación Profesional II 7

- Diplomatura 8

- Licenciatura 9

- Doctorado 10

- N.C. 99 